\title{
Constraining rapidly oscillating scalar dark matter using dynamic decoupling
}

\author{
Shahaf Aharony, ${ }^{1}$ Nitzan Akerman, ${ }^{2}$ Roee Ozeri, ${ }^{2}$ Gilad Perez, ${ }^{1}$ Inbar Savoray, ${ }^{1}$ and Ravid Shaniv ${ }^{2}$ \\ ${ }^{1}$ Department of Particle Physics and Astrophysics, Weizmann Institute of Science, Rehovot 7610001, Israel \\ ${ }^{2}$ Department of Physics of Complex Systems, Weizmann Institute of Science, Rehovot 7610001, Israel
}

(Received 2 April 2019; revised 11 December 2019; accepted 4 March 2021; published 20 April 2021)

We propose and experimentally demonstrate a method for detection of a light scalar dark matter (DM) field through probing temporal oscillations of fundamental constants in an atomic optical transition. Utilizing the quantum information notion of dynamic decoupling (DD) in a tabletop setting, we are able to obtain model-independent bounds on variations of $\alpha$ and $m_{e}$ at frequencies up to the $\mathrm{MHz}$ scale. We interpret our results to constrain the parameter space of light scalar DM field models. We consider the generic case, where the couplings of the DM field to the photon and the electron are independent, as well as the case of a relaxion DM model, including the scenario of a DM boson star centered around Earth. Given the particular nature of DD, allowing one to directly observe the oscillatory behavior of coherent DM and considering future experimental improvements, we conclude that our proposed method could be complimentary to, and possibly competitive with, gravitational probes of light scalar DM.

DOI: 10.1103/PhysRevD.103.075017

\section{INTRODUCTION}

The missing mass problem is one of the most fundamental questions in modern physics [1]. Although particle dark matter (DM) at the electroweak scale is a highly motivated solution [2], no discovery of such DM was made to date [3-5]. Another intriguing possibility is that of a sub-eV scalar DM field, coherently oscillating to account for the observed DM density (e.g., [6-8]). A coupling between the coherent DM candidate and the Standard Model (SM) particles would result in temporal oscillations of fundamental constants, such as the fine-structure constant and the electron's mass [6,8-14]. Here, we propose and experimentally demonstrate a method probing this DM signature in an atomic optical transition at a bandwidth ranging from a few $\mathrm{Hz}$ to the $\mathrm{MHz}$ range. This range, corresponding to a light scalar DM field which is coherently oscillating at these frequencies, has been a blind spot for current experimental measurements of time variations of fundamental constants (e.g., [15-17]), despite being theoretically motivated (e.g., $[7,18])$. Our proposal uses a tabletop setting and utilizes the quantum information notion of dynamic decoupling (DD) $[19,20]$ to amplify the desired signal within this uncovered bandwidth in a noisy environment [21].

Published by the American Physical Society under the terms of the Creative Commons Attribution 4.0 International license. Further distribution of this work must maintain attribution to the author(s) and the published article's title, journal citation, and DOI. Funded by SCOAP .
For a scalar field $\phi$, which couples to the electromagnetic field strength $F_{\mu \nu}$ and to the electron $e$ as [15,22]

$$
\mathcal{L}_{\text {int }} \supset \frac{g_{\phi \gamma}}{4} \phi F^{\mu \nu} F_{\mu \nu}-g_{\phi e} \phi \bar{e} e,
$$

the mass of the electron $m_{e}$ and the fine-structure constant $\alpha$ will be modified with respect to their SM values as

$$
\begin{aligned}
m_{e} & =m_{e}^{\mathrm{SM}}+\delta m_{e}, \quad \delta m_{e}=g_{\phi e}\langle\phi(t, \vec{x})\rangle, \\
\alpha & =\alpha^{\mathrm{SM}}+\delta \alpha, \quad \delta \alpha=g_{\phi \gamma}\langle\phi(t, \vec{x})\rangle \alpha^{\mathrm{SM}} .
\end{aligned}
$$

If $\phi$ is a DM candidate lighter than $\sim 0.1 \mathrm{eV}$, it is expected to have a large occupation number and is thus described as a classical field [8]. Therefore, $\phi$ will coherently oscillate with a frequency related to its mass $m_{\phi}$ and an amplitude that is determined by $m_{\phi}$ and the DM density $\rho_{\text {DM }}$ as [15]

$$
\langle\phi(t, \vec{x})\rangle \simeq \frac{\sqrt{2 \rho_{\mathrm{DM}}}}{m_{\phi}} \cos \left(m_{\phi}(t-\vec{v} \cdot \vec{x}+\ldots)\right),
$$

where $\vec{v}$ is the DM velocity. As a result, $\alpha$ and $m_{e}$ would undergo time-dependent modulations, which could be experimentally observed as will be explained below. Given experimental bounds on the variations of $\alpha$ and $m_{e}$ at a temporal modulation frequency $\nu[\mathrm{Hz}]=\mathrm{m}_{\phi} \mathrm{c}^{2} / \mathrm{h}$, denoted by $d_{m_{e}} \equiv\left(\frac{\delta m_{e}}{m_{e}}\right)^{\mathrm{UB}}$ and $d_{\alpha} \equiv\left(\frac{\delta \alpha}{\alpha}\right)^{\mathrm{UB}}$, we can set upper limits on the couplings of a DM candidate of the corresponding mass as 


$$
\begin{gathered}
g_{\phi e}\left(m_{\phi}\right) \leq d_{m_{e}}\left(m_{\phi}\right) m_{\phi} \frac{m_{e}}{\sqrt{2 \rho_{\mathrm{DM}}}}, \\
g_{\phi \gamma}\left(m_{\phi}\right) \leq d_{\alpha}\left(m_{\phi}\right) m_{\phi} \frac{1}{\sqrt{2 \rho_{\mathrm{DM}}}} .
\end{gathered}
$$

For a generic DM candidate, $g_{\phi e}$ and $g_{\phi \gamma}$ are independent. Experimental bounds for $d_{m_{e}}$ and $d_{\alpha}$ at a specific frequency can be obtained by monitoring oscillations of an atomic optical transition frequency that depends on $\alpha$ and on $m_{e}$, when compared to a frequency reference that depends differently on these parameters. However, these oscillations might be overshadowed by the noisy experimental environment. In order to amplify the desired signal while mitigating undesired noise, we propose to use DD [19].

\section{MEASURING TEMPORAL OSCILLATIONS OF FUNDAMENTAL CONSTANTS USING DD}

DD is a notion that utilizes the application of a known time-dependent Hamiltonian $\mathcal{H}(t)$ on an open quantum system in order to alter the effect of the environment on a specific subsystem. From a metrological point of view, $\mathcal{H}(t)$ functions as a spectral filter, screening the evolution of this subsystem outside of, and enhancing it in, an engineered spectral window.

Our experimental proposal relies on the comparison of the optical frequency of a trapped ion's optical clock transition to a narrow-linewidth laser locked to an ultrastable cavity and placing bounds on the amplitude of the ion-laser relative AC frequency shift at frequency $\nu$, denoted as $\Delta f(\nu)$. The experimental sequence is illustrated in Fig. 1. First, an optical $\frac{\pi}{2}$ laser pulse creates an equal superposition $\frac{1}{\sqrt{2}}(|g\rangle+|e\rangle)$ between the ion's ground state $|g\rangle$ and excited state $|e\rangle$. Next, laser $\pi$ pulses periodically rotate the ion's state around some equatorial axis on the Bloch sphere, flipping $|g\rangle$ and $|e\rangle$ at frequency of $2 \nu_{m}$. The resulting state is $\frac{1}{\sqrt{2}}\left(|g\rangle+e^{i \psi}|e\rangle\right)$, where $\psi$ is the superposition phase that depends on $\Delta f(\nu)$, the modulation at frequency $\nu_{m}$, and their relative phase $\xi$ (the function $\psi$ can be found in Appendix A). Information about $\psi$ can be obtained by applying a second interrogation laser $\frac{\pi}{2}$ pulse and scanning its phase relative to the initial $\frac{\pi}{2}$ pulse. This results in a Ramsey fringe. When $\nu=\nu_{m}$, the superposition phase acquired at each oscillation cycle is accumulated with experimental interrogation time, and the resulting Ramsey fringe exhibits a phase shift, which is maximal when $\xi=0$. This phase accumulation is possible under the assumption of a coherent DM field $\phi$, inducing a coherent oscillation of $\alpha$ and $m_{e}$. When $\nu \neq \nu_{m}$, the phase is not accumulated and is averaged to zero with experimental interrogation time, and therefore, the Ramsey fringe shows full contrast and negligible phase shift. When $\nu=\nu_{m}$, but $\xi$ is uniformly distributed between realizations, the resulting fringe is averaged over phase shifted Ramsey fringes, and

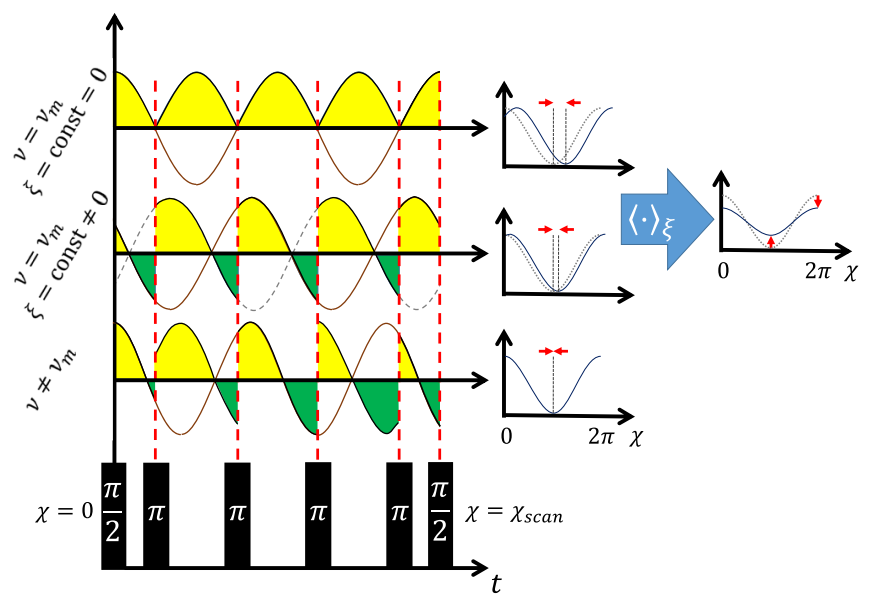

FIG. 1. Experimental sequence and form of measurements results. An example for a DD experimental sequence including four modulation $\pi$ pulses is illustrated at the bottom. The three time sequences above it show the ion superposition phase evolution at a single experimental realization for three cases, along with the corresponding Ramsey fringes. The dashed-line fringe corresponds to no fundamental constants oscillation, and the solid line fringe corresponds to the measured Ramsey fringe of the specific case. The time sequences show the ion superposition's rotation frequency (brown solid line) and phase accumulation (area under the function-yellow marks positive phase, and green marks negative phase) under fundamental constants oscillation at amplitude $\Delta f(\nu)$, frequency $\nu$, and phase relative to the pulse sequence of $\xi$.

$\Delta f(\nu)$ can be inferred from the fringe contrast reduction (right most fringe in Fig. 1). For a quantitative phase analysis, see Appendix A and [23]. By measuring either the Ramsey fringe phase $\psi$ in the synchronous case or the Ramsey fringe amplitude in the asynchronous case, for different $\nu_{m}$ values, bounds can be placed on the frequency oscillations amplitude $\Delta f\left(\nu_{m}\right)$ at each $\nu_{m}$ value. A detailed description of the above method can be found in [23].

The ion's transition frequency shift is proportional to the change in the Rydberg constant $R_{\infty} \propto \alpha^{2} m_{e}$, and therefore, the relative frequency change of the ion due to variations in $\alpha$ and $m_{e}$ is [24,25]

$$
\frac{\delta f_{\text {ion }}(\nu)}{f_{\text {ion }}}=2 \frac{\delta \alpha(\nu)}{\alpha}+\frac{\delta m_{e}(\nu)}{m_{e}} .
$$

In contrast, the laser's frequency shift is inversely proportional to the distance between the cavity mirrors $r_{0}$, which is proportional to the Bohr radius $a_{0} \propto\left(\alpha m_{e}\right)^{-1}[9,17]$. Therefore, the relative frequency change of the laser would depend on $\alpha$ and $m_{e}$ as

$$
\frac{\delta f_{\text {laser }}(\nu)}{f_{\text {laser }}}=\left(\frac{\delta \alpha(\nu)}{\alpha}+\frac{\delta m_{e}(\nu)}{m_{e}}\right) \times F(\nu) .
$$

Here, $F(\nu)$ denotes a frequency-dependent response of the laser frequency to the change in $a_{0}$ at a specific signal 
frequency $\nu$. At frequencies much lower than the cavity's lowest mechanical mode and optical linewidth, $r_{0}$ follows the change in $a_{0}$, and the laser changes its frequency accordingly, meaning $F(\nu=$ low $) \rightarrow 1$. At frequencies much higher than the cavity's linewidth and the ratio between the speed of sound in the cavity $v_{\text {sound }}$ and $r$, the cavity's mechanical response to the variations in $a_{0}$ is lowpass filtered, and, in addition, the laser cannot follow the cavity's instantaneous frequency due to the finite lifetime of a photon in the cavity. Therefore, the laser frequency response to variations in $a_{0}$ is further reduced, and $F(\nu=$ high $) \rightarrow 0$. Assuming $f_{\text {cavity }} \approx f_{\text {ion }}=f_{0}$, we obtain

$$
\begin{aligned}
\frac{\Delta f(\nu)}{f_{0}} & =\frac{\delta f_{\text {ion }}(\nu)-\delta f_{\text {laser }}(\nu)}{f_{0}} \\
& =(2-F(\nu)) \frac{\delta \alpha}{\alpha}+(1-F(\nu)) \frac{\delta m_{e}}{m_{e}},
\end{aligned}
$$

leading to the conclusion that at low frequencies, only $\alpha$ variation is detectable, whereas at high frequencies, variations in both constants may be observed.

Below, we present bounds on DM obtained from a proofof-principle measurement, in which we used a laser at $674 \mathrm{~nm}$ locked to a $r=0.1 \mathrm{~m}$ long, high-finesse $(300,000)$ ultralow expansion (ULE) optical Fabri-Pérot cavity, with a $4.5 \mathrm{kHz}$ linewidth. This laser frequency matched the clock dipole-transition $5 S_{\frac{1}{2}} \leftrightarrow 4 D_{\frac{5}{2}}$ of a single ${ }^{88} \mathrm{Sr}^{+}$ion, on which, the DD sequence was applied. Here, $\nu_{m}=1013 \mathrm{~Hz}$ was chosen. The difference between the superposition phase $\phi$ and the laser phase was mapped onto the populations of the ground and excited states by applying a final interrogation $\frac{\pi}{2}$ laser pulse at pulse phase $\chi$, with respect to the initial $\frac{\pi}{2}$ pulse, and scanning $\chi$ between 0 and $2 \pi$ (see Fig. 1). Assuming no synchronization between the control optical pulses modulation phase and the optical frequency oscillation at $\nu_{m}$, a bound for the transition frequency modulation amplitude was readily inferred from the deviation of the resulting Ramsey fringe amplitude from 0.5 (see Fig. 1 and Appendix A). More details about the experimental parameters and setup can be found in [23].

The upper bound for the relative frequency modulation amplitude at different values of $\nu$ is given in Fig. 2. The sharp peaks appear at frequencies where the experimental DD sequence loses sensitivity (see Appendix A). The best sensitivity is obtained for $\nu=\nu_{m}$ and, by scanning this frequency high sensitivity, can be maintained for the entire scan range. The dash-dotted line in Fig. 2 is the expected bound obtained from performing the experiment proposed above for different values of $\nu_{m}$ and assuming one measures the same Ramsey fringe amplitude as for $\nu_{m}=1013 \mathrm{~Hz}$. The bound on $\Delta f / f_{0}$ corresponds to bounds on $d_{\alpha}$ or $2 d_{\alpha}+d_{m_{e}}$, as explained above and shown in the plot. While stricter constraints on these parameters already exist in the literature [16,26-28], our bound is the first one

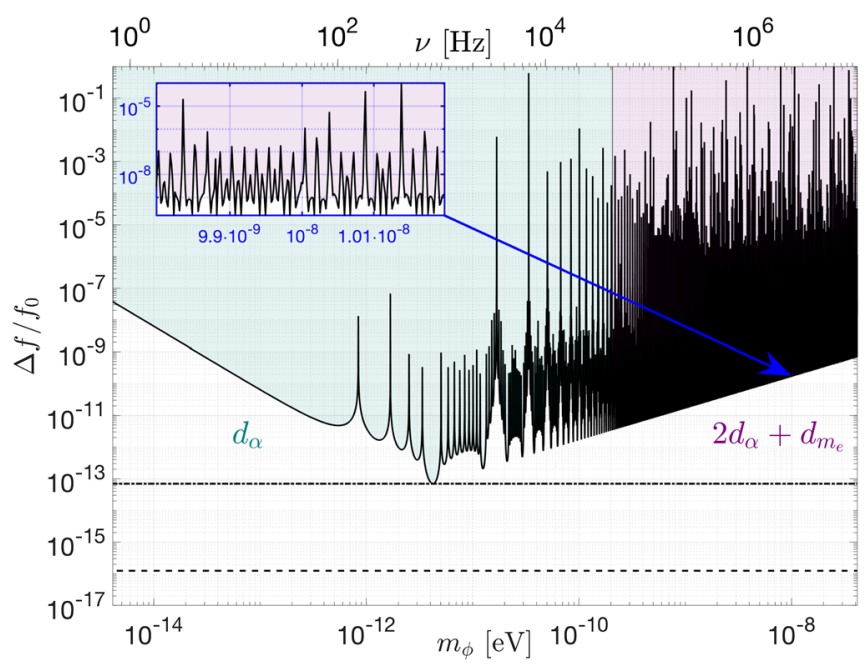

FIG. 2. Bounds on the relative modulation of the transition frequency from a DD experiment, placed at 95\% C.L. The solid black line marks the current bound, obtained by a measurement at a single modulation frequency point $\nu_{m}=1013 \mathrm{~Hz}$. The dasheddotted line marks the current sensitivity reach, corresponding to scanning over $\nu_{m}$. The dashed line marks the future-projected sensitivity, considering fundamental effects and an averaging time of 30 days per frequency point. The inset is a magnified view of $m_{\phi} \sim 10^{-8} \mathrm{eV}$.

directly, and model independently, constraining temporal oscillations of $\alpha$ and $m_{e}$ in the $10 \mathrm{~Hz}-\mathrm{MHz}$ frequency range.

The current bound is mostly limited by experimental imperfections and can be improved by at least 3 orders of magnitude. We estimate the future-projected bound taking into account only fundamental effects, i.e., ion decay time and quantum projection noise, and considering an averaging time of 30 days per frequency scan point. The future-projected bound is shown as a dashed line in Fig. 2. We also note that in the current setup, the signal was encoded in the coherence of the ion's superposition or, alternatively, the amplitude of the Ramsey fringe [23]. Since both the signal and the experimental imperfections (e.g., $\pi$ pulse fidelity) tend to decrease the fringe amplitude, the bound would be ultimately limited by the experimental apparatus. However, in the case of large enough quality factor of the $\delta m_{e}$ and $\delta \alpha$ oscillations, it would be useful to synchronize different experiment realizations via an external clock, such that, for a specific $\nu_{m}$, different experimental realizations would measure signal oscillations with the same $\xi[29,30]$. This would allow one to infer the signal amplitude from the final superposition phase, separating it from the coherence of our atom (see Fig. 1).

\section{BOUNDS ON LIGHT SCALAR DARK MATTER FROM DD EXPERIMENTS}

We are interested in the implications of our measurements to models of light scalar DM. Using the results shown in Fig. 2, we obtain upper limits on the values of $g_{\phi \gamma}$ and $g_{\phi e}$ at $95 \%$ C.L. and present them in Fig. 3(a) 
and Fig. 3(b), respectively. The background DM density is assumed to be $\rho_{\mathrm{DM}}=\rho_{\mathrm{DM}_{\odot}}=3.1 \times 10^{-6} \mathrm{eV}^{4}$, which is the local DM density around the Sun [31]. For our analysis, we assumed a sharp transition between $d_{\alpha}$ sensitivity and $d_{m_{e}}+2 d_{\alpha}$ sensitivity at $\nu_{\text {step }}=50 \mathrm{kHz}$, namely $F(\nu)=$ $\Theta(\nu-50 \mathrm{kHz})$, where $\Theta$ is the Heaviside step function. The step frequency was chosen to be the ratio between the speed of sound in our cavity spacer $v_{\text {sound }} \approx 5 \frac{\mathrm{km}}{\mathrm{s}}$ and the cavity length $r \approx 0.1 \mathrm{~m}$. The optical linewidth is an order of magnitude lower. The black dashed line represents the future-projected sensitivity for the proposed method, corresponding to scanning the experimental modulation frequency $\nu_{m}$. We compare our results with the current bounds obtained from experimental tests of free fall universality. We also highlight the boundary of the region favored by Naturalness criteria (see, e.g., $[8,15,32,33])$. Both are explained below.

We further interpret our results in accordance to the relaxion [34] model, which was recently shown to be a viable DM candidate [7]. The interactions of a relaxion DM with the SM fields are mediated through its mixing with the Higgs, and thus, the corresponding couplings are no longer independent. The couplings of the relaxion to the electron and the photon are given by [22]

$$
\begin{aligned}
& g_{\phi e}=Y_{e} \sin \theta, \\
& g_{\phi \gamma}=-\frac{\alpha_{0} \sin \theta}{2 \pi v}\left|A_{W}\left(\tau_{W}\right)+\sum_{\text {fermions }} N_{c, f} Q_{f}^{2} A_{F}\left(\tau_{f}\right)\right|,
\end{aligned}
$$

where $Y_{e}$ is the Yukawa coupling of the electron to the Higgs $(h), \theta$ is the mixing angle between the relaxion and the Higgs, and $\tau_{x}=m_{h}^{2} / 4 m_{x}^{2} . A_{F}(\tau)$ and $A_{W}(\tau)$ are defined in [22] and calculated accordingly. The upper bound on $\Delta f / f_{0}$ can then be used to exclude the region in the $m_{\phi}-\sin (\theta)$ parameter space corresponding to

$$
\sin \theta \leq \frac{\left(\frac{\Delta f}{f_{0}}\right)^{\mathrm{UB}} \times \ldots}{\sqrt{2 \rho_{\mathrm{DM}_{\odot}}}\left(\frac{\sqrt{2} \kappa_{e}}{v}(1-F)-1.12 \times 10^{-14}(2-F)\right)},
$$

where $\kappa_{e} \equiv Y_{e} / Y_{e}^{\mathrm{SM}}$. Analyzing the results in Fig. 2 for relaxion DM, we obtain the appropriate upper limit on $\sin \theta$, assuming $\kappa_{e}=1$, and present it in Fig. 3c. Note that the current upper bound on $\kappa_{e}$ is at 610 [35], which would yield a stronger constraint. The analysis presented here can also be modified to apply to other Higgs portal DM scenarios [36].

Moreover, relaxions can form what are known as boson stars [37], which could be bound to the Earth's gravitational potential [38]. Although the formation process of such objects is still an open question, if formed-they should be stable as they are the ground state of the gravitational

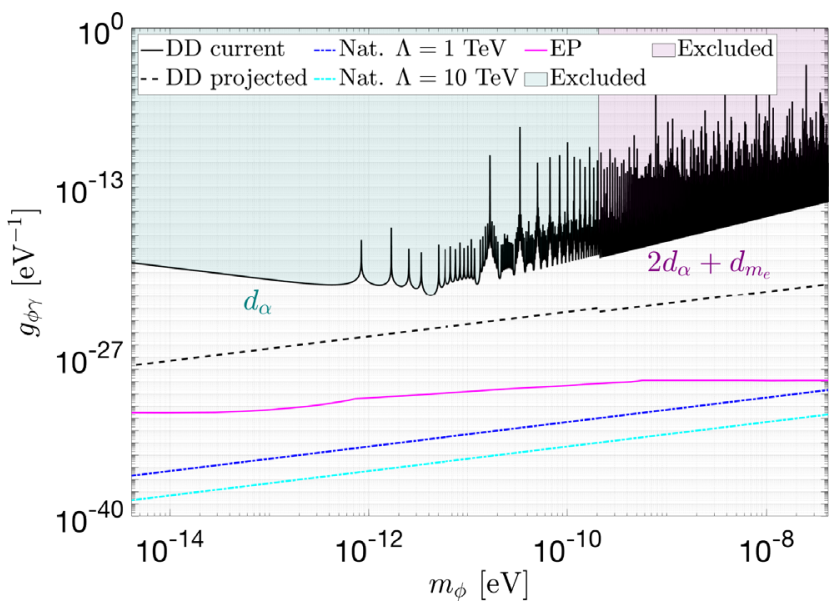

(a)

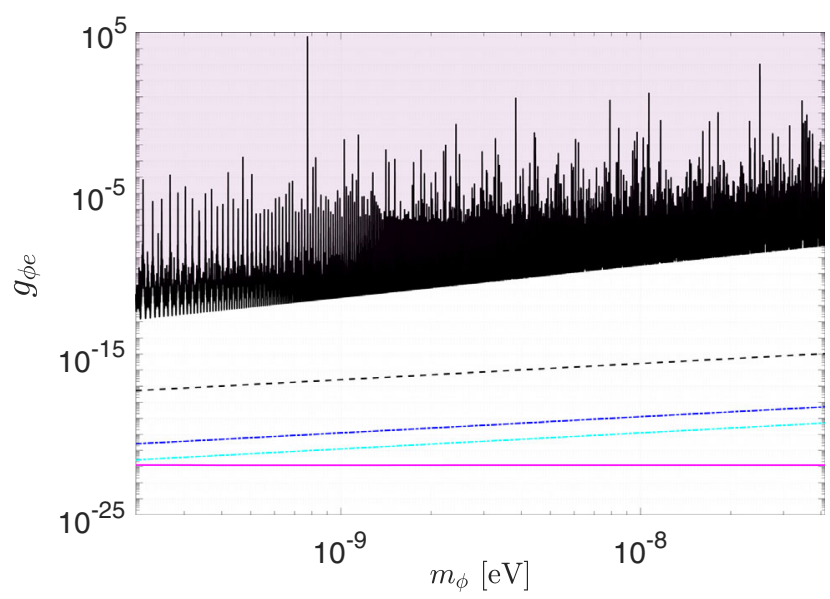

(b)

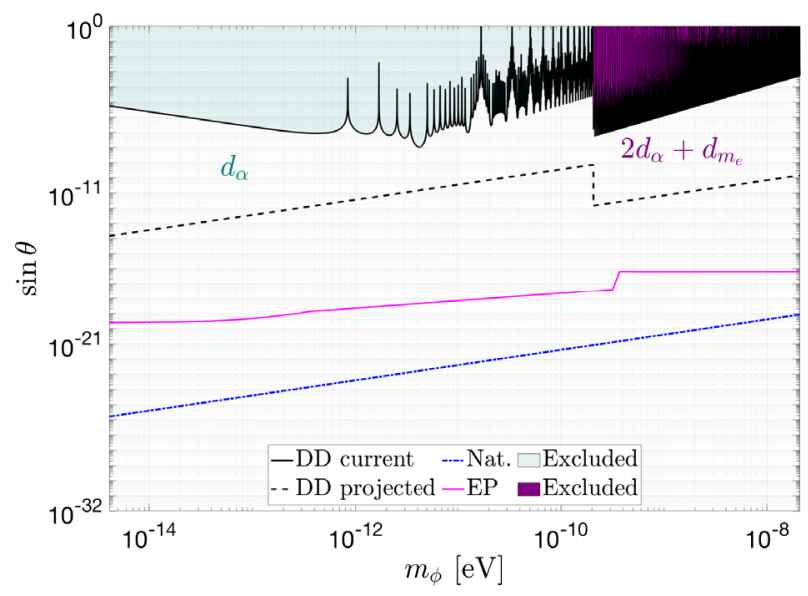

(c)

FIG. 3. Bounds on the parameter space of light scalar DM corresponding to the observed DM density near the Sun. The bounds on the couplings of a generic DM candidates are shown in (a) and (b). The bounds on the mixing angle of a relaxion DM are presented in (c). Black: current and projected bounds from DD experiments at 95\% C.L. Magenta: EP-test bounds taken from [26]. Blue and cyan dash-dotted: Naturalness criteria. 
system [38]. The presence of the DM star will increase the DM density around Earth at all times and, accordingly, the signal measured in our proposed experiment. The mass of the relaxion star is constrained by local measurements of gravitational acceleration [39] and should satisfy $M_{\star} \lesssim 4 \times 10^{-9} M_{\oplus}$, where $M_{\oplus}$ is the mass of the Earth. Using the star density profile presented in [38] as the DM density, combined with the bounds shown in Fig. 2, we obtain an upper limit on $\sin \theta$ and present it in Fig. 4. The allowed parameter region for relaxion DM is also indicated [7]. A corresponding bound on $g_{\phi \gamma}$ can be found in Appendix B.

A new light scalar coupled to SM fields would introduce a potential between otherwise neutral test masses (see, e.g., $[40,41])$. Therefore, another constraint on the parameter space of a light scalar DM arises from experiments testing the equivalence principle (EP), designated to detect deviations from gravity. The bounds related to EP tests presented here are based on those given in [26]. The most stringent constraint on the relaxion model, in this context, is coming from its coupling to the gluon via $g_{\phi g} \sim \alpha_{s} \sin \theta / 3 \pi v$, as given in $[22,42]$. Although the bounds we obtain from DD are currently weaker than those set by the experimental tests of gravity, they are different in essence. The constraints resulting from DD experiments are precisely related to the temporal variations of $\alpha$ and $m_{e}$, whereas EP tests are unable to directly measure time-dependent phenomena. This difference would be important in the case of a discovery of a rapidly oscillating scalar DM. While gravitational tests could only indicate a possible candidate, our proposed method could also immediately observe its oscillatory nature and thus, positively identify it as a

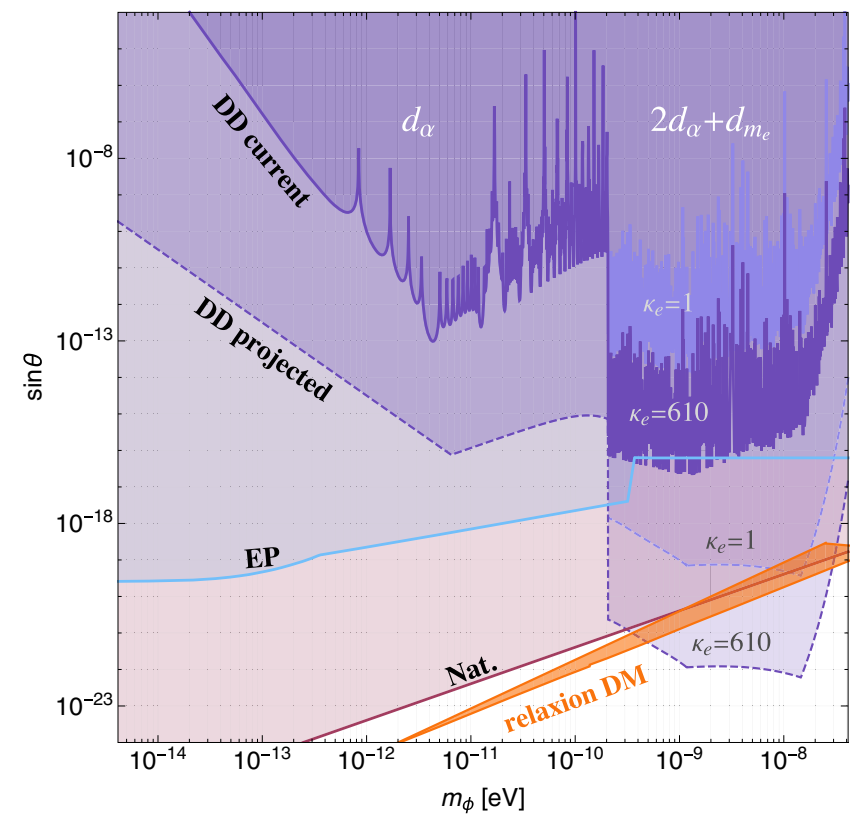

FIG. 4. Bounds for a DM star centered around Earth, along with the theoretically motivated region for a relaxion DM given in [7]. coherent DM field. In addition, it can be seen that for some region of the parameter space, our future-projected bounds could become competitive with those of gravitational tests for the scenario of a DM star, even before fully exhausting the experimental improvements suggested above. Finally, it should be noted that, unlike EP tests, our experiment is sensitive to the DM density close to earth, and so combining it with gravitational tests could probe the three-dimensional parameter space $m_{\phi}-\sin \theta-\rho_{\mathrm{DM}}^{\oplus}$.

Since $\phi$ is a scalar field, its mass parameter is sensitive to radiative corrections resulting from its interactions. To maintain naturalness, its quantum corrections should be small compared to the bare mass $\delta m_{\phi_{1-\text { loop }}}^{2} \ll m_{\phi_{\text {bare }}}^{2}$. For a scalar field with the interaction terms describes above, this would imply $[8,15,32]$

$$
\left|g_{\phi e}\right| \ll \frac{4 \pi m_{\phi}}{\Lambda}, \quad\left|g_{\phi \gamma}\right| \ll \frac{16 \pi m_{\phi}}{\Lambda^{2}} .
$$

For a relaxion DM, the condition is $\sin \theta \leq \frac{m_{\phi}}{v}$ [33].

\section{CONCLUSION}

Rapidly oscillating scalar DM field is a well-motivated scenario but currently lies in a blind spot of existing experimental searches sensitive to coherent oscillations of fundamental constants. In this paper, we have proposed a new experimental probe of light scalar DM, utilizing the method of DD in a tabletop setting. Using a proof-ofconcept experimental measurement, we have obtained model-independent bounds on the temporal oscillations of both $m_{e}$ and $\alpha$ at frequencies up to the $\mathrm{MHz}$ scale. Consequently, we were able to set upper limits on the couplings of a generic coherent DM candidate. We have also interpreted the results for the case of relaxion DM, for which, our constraints are significantly tightened. As an experimental outlook, we believe that the bounds presented here can be improved significantly in two ways. First, the modulation frequency $\nu_{m}$ can be scanned, and therefore, a lower bound can be achieved for a range of frequencies. Second, instead of the superposition coherence, its phase shift can be measured by synchronizing different experimental realizations, separating the desired signal from unwanted experimental imperfections. Therefore, our proposed method could be an important tool for studying light scalar DM, not only directly probing its oscillatory nature, but also possibly setting constraints that would be competitive with EP tests and that would probe the theoretically motivated parameter space of a relaxion DM. Additional measurements covering complementary parts of the spectrum have been recently concluded, and their reports were in preparation at the time this manuscript was being considered for publication $[43,44]$. 


\section{ACKNOWLEDGMENTS}

We would like to acknowledge early discussions on these ideas with A. Derevianko, S. Kolkowitz, and D. Budker. We thank D. Budker and Y. Nir for comments on the manuscript. We are grateful to J. A. Eby and H. Kim for the assistance and the advice regarding relaxion stars. R. O. and R.S. acknowledge support by the Crown Photonics Center, ICORE-Israeli excellence center Circle of Light, The Israeli Science Foundation, the Israeli Ministry of Science Technology and Space and the European Research Council (consolidator grant 616919-Ionology). The work of G. P. is supported by grants from the BSF, ERC, ISF; the work of R. O. and G. P. is jointly support by the Minerva Foundation, the German-Israeli Foundation (GIF), and the Serge Research Award. I. S. is supported by a fellowship from the Ariane de Rothschild Women Doctoral Program.

\section{APPENDIX A: SENSITIVITY AND FILTER FUNCTION}

Applying optical $\pi$ pulses in a repeating unit cell of

[wait time $\tau]-[\pi$ pulse $]-[$ wait time $\tau]$

results in a phase modulation kernel of the form,

$$
\begin{aligned}
f(t, \tau, n)= & \operatorname{rect}\left(\frac{t}{2 n \tau}\right) \\
& \times\left[\Theta(t)+2 \sum_{k=1}^{\infty}(-1)^{k} \Theta(t-(2 k-1) \tau)\right],
\end{aligned}
$$

where $\Theta$ is the Heaviside step function, $n$ is the number of pulses, and rect is a rectangular window function nulling the modulation at $t<0$ and $t>2 n \tau$. $\tau$ defines the frequency $\nu_{m}$ from the main text by $\tau=\frac{1}{4 \nu_{m}}$. We assume an oscillating atomic angular frequency detuning in the form of

$$
\delta(t, \xi)=2 \pi \Delta f \sin (2 \pi \nu t+\xi),
$$

attributed, for instance, to an oscillating DM field. Here, $\Delta f$ is the detuning amplitude, namely the amplitude of the time dependent atomic frequency shift, and $\xi$ is the phase mismatch between the signal and the pulse modulation. The resulting superposition phase $\psi$ accumulated over the pulse modulation sequence is, therefore,

$$
\begin{aligned}
\psi(\tau, n, \xi) & =\int_{-\infty}^{\infty} f(t, \tau, n) \delta(t, \xi) d t \\
& =4 \frac{\Delta f}{\nu} \times \ldots \\
& \frac{\cos \left(2 \pi \nu n \tau+\xi+n \frac{\pi}{2}\right) \sin \left(2 \pi \nu n \tau-n \frac{\pi}{2}\right) \sin ^{2}\left(\frac{2 \pi \nu \tau}{2}\right)}{\cos (2 \pi \nu \tau)} .
\end{aligned}
$$

This would be the signal corresponding to a phase estimation experiment. In the experimental bound presented in this work, the theoretical fringe amplitude $A$ takes the form of

$$
A(\tau, n)=0.5\left|\langle\cos (\psi(\tau, n, \xi))\rangle_{\xi}\right|,
$$

where $\langle\cdot\rangle_{\xi}$ denotes averaging over $\xi$ 's sampled from a uniform distribution between 0 and $2 \pi$. The resulting contrast is given by

$A(\tau, n)=0.5\left|J_{0}\left(4 \frac{\Delta f}{\nu} \times \frac{\sin (2 \pi \nu n \tau) \sin ^{2}\left(\frac{2 \pi \nu \tau}{2}\right)}{\cos (2 \pi \nu \tau)}\right)\right|$,

where $J_{0}$ is the zeroth Bessel function of the first kind. This function was used to obtain the bounds on the detuning amplitude $\Delta f$ presented in Fig. 2. As temporal oscillations of fundamental constants would result in the reduction of the contrast, an upper limit on $\Delta f$ corresponds to the 95\% C.L. lower limit on $A$. Since the lower bound on $A$ was found to be close to the global maximum of $J_{0}$, Eq. (A5) yields a unique solution for $\Delta f$ for each frequency $\nu$. The functional behavior of this bound is then fully determined by the argument of the Bessel function above.

In the presented experimental bound, we believe that experimental imperfections, such as pulse time and phase imperfections, are the limiting factors that set the lowest DM bound. We now turn to present the analysis for the projected bound of the experiment (dashed line in Fig. 2), which is about 3 orders of magnitude stronger. We calculate the expected bound on the frequency oscillation amplitude, for a 390 ms Ramsey interrogation time, equal to our ion, ${ }^{88} \mathrm{Sr}^{+}$, exponential decay constant. Here, we assume no external noises, other than projection noise, and no reduction in contrast other than from the lifetime decay.

First, we analyze the parametric uncertainty of our measurement, for DM frequency oscillation amplitude $\Delta f$. We assume Ramsey time $T=2 n \tau$, decay time $\tau_{\mathrm{dec}}$, and total experimental interrogation time of $\tau_{\text {tot }}$. After the Ramsey wait time, we get a spontaneous decay contrast reduction of $\exp \left\{-T / \tau_{\mathrm{dec}}\right\}$, meaning we have $p=1 / 2+$ $\exp \left\{-T / \tau_{\text {dec }}\right\} \cos \phi A(\tau, n)$ as the Bernoulli parameter, where $\phi$ is the angle difference between the two Ramsey $\pi / 2$ pulses, and $A(\tau, n)$ is the contrast function (A5). From now on, we will omit the phase $\phi$ because we can assume that we measure at the point of highest contrast signal and lowest noise $(\phi=0, \pi)$.

For $\nu \rightarrow 1 / 4 \tau$, which means we are using this contrast to place bound on frequency oscillation with amplitude $\Delta f$ and frequency on resonance with our DD sequence, we get the contrast expression,

$$
|A(\tau, n)|_{\nu \rightarrow 1 / 4 \tau}=\frac{1}{2}\left|J_{0}(8 \Delta f T)\right|,
$$

where $J_{0}$ is the zeroth Bessel function. We can now write

$$
p=\frac{1}{2}+\frac{1}{2} e^{-\frac{T}{\tau_{\mathrm{dec}}}}\left|J_{0}(8 \Delta f T)\right|
$$


Next, we assume that, in reality, the DM effect is vanishingly small (meaning $\Delta f=0 \rightarrow\left|J_{0}(8 \Delta f T)\right|=0$ ), but we have some statistical uncertainty for the contrast we measure. For sufficiently large number of measurements $N$, this uncertainty goes as

$$
\sigma_{p}=\sqrt{\frac{p(1-p)}{N}} .
$$

We would like to place a bound on the deviation of our averaged measured contrast from the expected contrast resulting from the decay lifetime. Our measurement result would fall within $95 \%$ confidence between 1 (full contrast) and 2 standard deviations away:

$$
\frac{1}{2}+\frac{1}{2} e^{-\frac{T}{\tau_{\mathrm{dec}}}}-2 \sigma_{p} \leq \text { result } \leq \frac{1}{2}+\frac{1}{2} e^{-\frac{T}{\tau_{\mathrm{dec}}}}+2 \sigma_{p} .
$$

The lower bound defines the maximal frequency modulation amplitude that might hide in the statistics. We, therefore, assume that any deviation from only spontaneous decay in our measurement is due to DM oscillation and write

$$
\frac{1}{2}+\frac{1}{2} e^{-\frac{T}{\tau_{\mathrm{dec}}}}\left|J_{0}\left(8 \Delta f_{\mathrm{ub}} T\right)\right|=\frac{1}{2}+\frac{1}{2} e^{-\frac{T}{\tau_{\mathrm{dec}}}}-2 \sigma_{p},
$$

where $\Delta f_{\text {ub }}$ is the upper bound for the frequency modulation. For small (even vanishing) $\Delta f_{\text {ub }}$, we get $\left|J_{0}\left(8 \Delta f_{\mathrm{ub}} T\right)\right| \sim 1-4\left(\Delta f_{\mathrm{ub}} T\right)^{2}$, where we used the Taylor series of the zeroth order Bessel function. We can, therefore, write

$\Delta f_{\mathrm{ub}}=\frac{1}{T} \sqrt{e^{-\frac{T}{\tau_{\mathrm{dec}}}} \sigma_{p}}=\frac{1}{T} \sqrt{\frac{1}{2} e^{-\frac{T}{\tau_{\mathrm{dec}}}} \sqrt{\frac{T\left(1-e^{-\frac{2 T}{\tau_{\mathrm{dec}}}}\right.}{\tau_{\mathrm{tot}}}}}$,

where we write the number of measurements as $N=\tau_{\text {tot }} / T$.

From this expression, we can find the optimal value of $T$, for which, $\Delta f_{\mathrm{ub}}$ is the smallest. Assuming 30 days of averaging, which amounts to $\tau_{\text {tot }}=2592000 \mathrm{sec}$ and using $\tau_{\mathrm{dec}}=0.39 \mathrm{sec}$, we get that the optimal $T=0.55 \mathrm{sec} \sim$ $\sqrt{2} \tau_{\text {dec }}$. The plot of $\Delta f_{\text {ub }}$ as a function of the Ramsey time $T$ is presented in Fig. 5. The resulting expected bound is calculated to be $\Delta f_{\text {ub }}=0.039$, which, in terms of relative frequency, amounts to

$$
\Delta f_{\mathrm{ub}} / f=0.055 /\left(4.4 \times 10^{14}\right) \sim 1.24 \times 10^{-16} .
$$

In addition to the demonstrated experiment, we proposed a synchronized experiment, in which the modulation is synchronized to an external clock, and instead of measuring the Ramsey fringe contrast $A$ corresponding to the superposition coherence, one would measure the Ramsey fringe phase $\psi$. The advantage of this scheme is that the signal will scale linearly with $T$ (as opposed to the quadratic dependent in case of contrast reduction), and, in addition, some of the experimental imperfections effects can be separated from the desired signal. The phase after a single

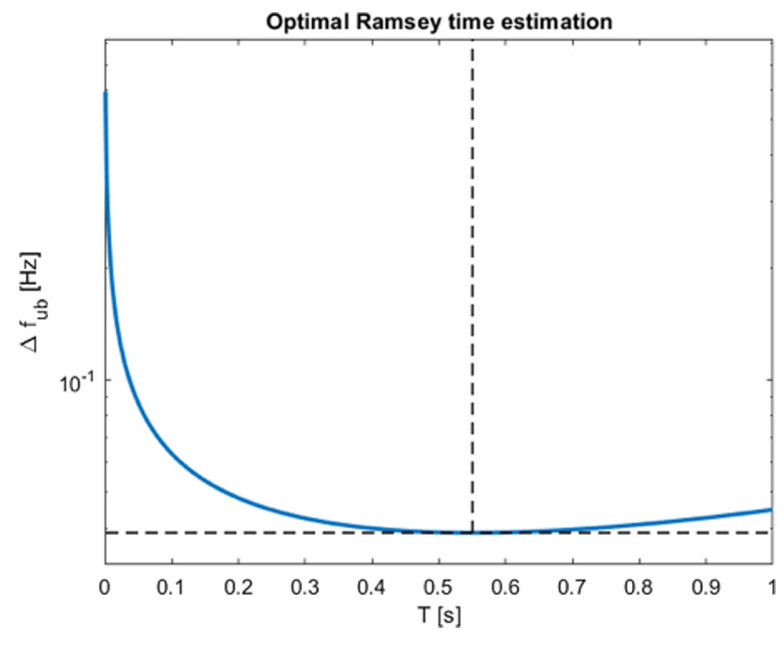

FIG. 5. The expected bound for the amplitude $\Delta f$ as a function of the Ramsey time $T$.

DD experimental realization with interrogation time $T$ looking for signal at $\nu_{m}=\nu$ would have the form,

$$
|\psi(T, \xi)|=4 T \Delta f \times|\cos (\xi)| .
$$

Within the DM oscillation coherence time, the phase $\xi$ can be scanned in different realizations. The maximal phase $4 T \Delta f$ would be obtained for $\xi=0$. Therefore, the sensitivity for measuring $\Delta f$ scales as $\frac{1}{T}$.

\section{APPENDIX B: RELAXION STAR}

A bound on the coupling of a DM candidate to photons $g_{\phi \gamma}$ is presented in Fig. 6 for a DM star bounded to earth.

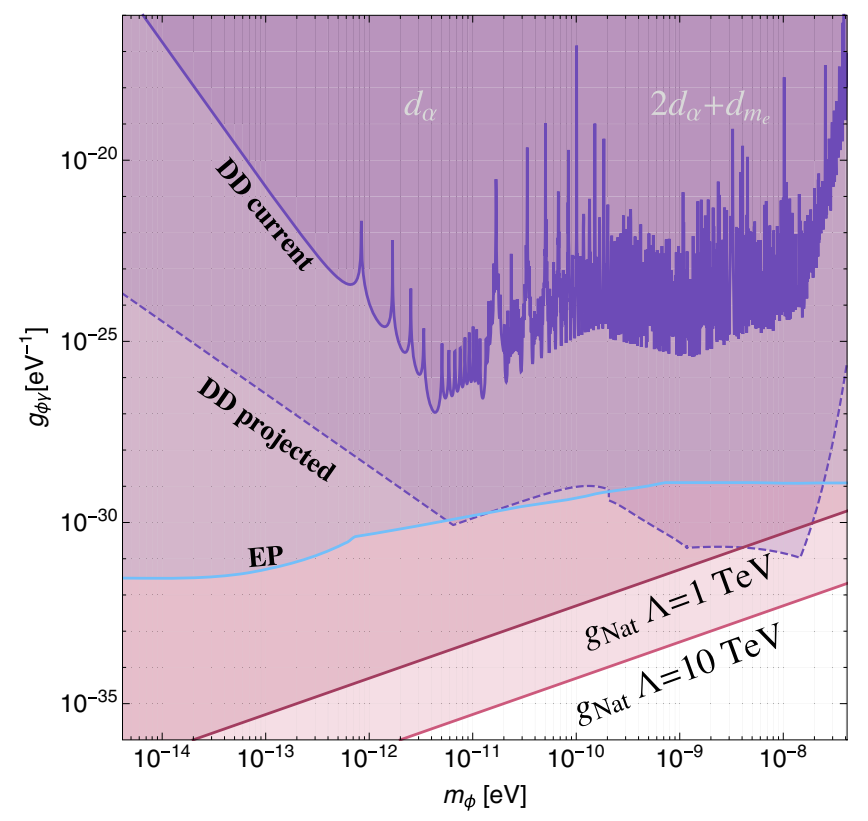

FIG. 6. Bound on $g_{\phi \gamma}$ for a relaxion star centered around Earth. 
[1] G. Bertone and D. Hooper, Rev. Mod. Phys. 90, 045002 (2018).

[2] G. Jungman, M. Kamionkowski, and K. Griest, Phys. Rep. 267, 195 (1996).

[3] G. Bertone, D. Hooper, and J. Silk, Phys. Rep. 405, 279 (2005).

[4] C. F. P. da Silva (LUX Collaboration), arXiv:1710.03572.

[5] E. Aprile et al. (XENON Collaboration), Phys. Rev. Lett. 121, 111302 (2018).

[6] A. Arvanitaki, J. Huang, and K. Van Tilburg, Phys. Rev. D 91, 015015 (2015).

[7] A. Banerjee, H. Kim, and G. Perez, Phys. Rev. D 100, 115026 (2019).

[8] P. W. Graham, D. E. Kaplan, J. Mardon, S. Rajendran, and W. A. Terrano, Phys. Rev. D 93, 075029 (2016).

[9] Y. V. Stadnik and V. V. Flambaum, Phys. Rev. Lett. 114, 161301 (2015).

[10] Y. V. Stadnik and V. V. Flambaum, Phys. Rev. Lett. 115, 201301 (2015).

[11] M. S. Safronova, D. Budker, D. DeMille, D. F. J. Kimball, A. Derevianko, and C. W. Clark, Rev. Mod. Phys. 90, 025008 (2018).

[12] B. M. Roberts, G. Blewitt, C. Dailey, M. Murphy, M. Pospelov, A. Rollings, J. Sherman, W. Williams, and A. Derevianko, Nat. Commun. 8, 1195 (2017).

[13] A. Derevianko and M. Pospelov, Nat. Phys. 10, 933 (2014).

[14] A. Derevianko, Phys. Rev. A 97, 042506 (2018).

[15] A. Arvanitaki, S. Dimopoulos, and K. Van Tilburg, Phys. Rev. Lett. 116, 031102 (2016).

[16] K. Van Tilburg, N. Leefer, L. Bougas, and D. Budker, Phys. Rev. Lett. 115, 011802 (2015).

[17] A. A. Geraci, C. Bradley, D. Gao, J. Weinstein, and A. Derevianko, Phys. Rev. Lett. 123, 031304 (2019).

[18] B. Döbrich, J. Phys. Conf. Ser. 632, 012004 (2015).

[19] L. Viola, E. Knill, and S. Lloyd, Phys. Rev. Lett. 82, 2417 (1999).

[20] S. Kotler, N. Akerman, Y. Glickman, A. Keselman, and R. Ozeri, Nature (London) 473, 61 (2011).

[21] We acknowledge early discussions on this idea with Andrei Derevianko and Shimon Kolkowitz.

[22] T. Flacke, C. Frugiuele, E. Fuchs, R. S. Gupta, and G. Perez, J. High Energy Phys. 06 (2017) 050.

[23] R. Shaniv and R. Ozeri, Nat. Commun. 8, 14157 (2017).
[24] P. Wcisło, P. Morzyński, M. Bober, A. Cygan, D. Lisak, R. Ciuryło, and M. Zawada, Nat. Astron. 1, 0009 (2016).

[25] Y. V. Stadnik and V. V. Flambaum, Phys. Rev. A 93, 063630 (2016).

[26] A. Hees, O. Minazzoli, E. Savalle, Y. V. Stadnik, and P. Wolf, Phys. Rev. D 98, 064051 (2018).

[27] P. Wcisło, P. Ablewski, K. Beloy, S. Bilicki, M. Bober, R. Brown, R. Fasano, R. Ciuryło, H. Hachisu, T. Ido, J. Lodewyck, A. Ludlow, W. McGrew, P. Morzyński, D. Nicolodi, M. Schioppo, M. Sekido, R. Le Targat, P. Wolf, X. Zhang, B. Zjawin, and M. Zawada, Sci. Adv. 4 (2018).

[28] R. M. Godun, P. B. R. Nisbet-Jones, J. M. Jones, S. A. King, L. A. M. Johnson, H. S. Margolis, K. Szymaniec, S. N. Lea, K. Bongs, and P. Gill, Phys. Rev. Lett. 113, 210801 (2014).

[29] S. Schmitt, T. Gefen, F. M. Stürner, T. Unden, G. Wolff, C. Müller, J. Scheuer, B. Naydenov, M. Markham, S. Pezzagna et al., Science 356, 832 (2017).

[30] J. M. Boss, K. Cujia, J. Zopes, and C. L. Degen, Science 356, 837 (2017).

[31] P. Salucci, F. Nesti, G. Gentile, and C. F. Martins, Astron. Astrophys. 523, A83 (2010).

[32] S. Dimopoulos and G. Giudice, Phys. Lett. B 379, 105 (1996).

[33] C. Frugiuele, E. Fuchs, G. Perez, and M. Schlaffer, J. High Energy Phys. 10 (2018) 151.

[34] P. W. Graham, D. E. Kaplan, and S. Rajendran, Phys. Rev. Lett. 115, 221801 (2015).

[35] A. Dery, C. Frugiuele, and Y. Nir, J. High Energy Phys. 04 (2018) 044.

[36] F. Piazza and M. Pospelov, Phys. Rev. D 82, 043533 (2010).

[37] M. Colpi, S. L. Shapiro, and I. Wasserman, Phys. Rev. Lett. 57, 2485 (1986).

[38] A. Banerjee, D. Budker, J. Eby, H. Kim, and G. Perez, Commun. Phys. 3, 1 (2020).

[39] S. L. Adler, J. Phys. A 41, 412002 (2008).

[40] J. E. Moody and F. Wilczek, Phys. Rev. D 30, 130 (1984).

[41] S. Dimopoulos and G. Giudice, Phys. Lett. B 379, 105 (1996).

[42] A. Djouadi, Phys. Rep. 457, 1 (2008).

[43] C. J. Kennedy, E. Oelker, J. M. Robinson, T. Bothwell, D. Kedar, W. R. Milner, G. E. Marti, A. Derevianko, and J. Ye, Phys. Rev. Lett. 125, 201302 (2020).

[44] D. Antypas, O. Tretiak, A. Garcon, R. Ozeri, G. Perez, and D. Budker, Phys. Rev. Lett. 123, 141102 (2019). 